\title{
Research and Application of Mathematical Model of Transmission Grating Signal Lissajous Figure
}

\author{
Luqing $\mathrm{Hu}^{1}$, Xianqing Lei ${ }^{1}$, Xiaoyi Wang ${ }^{1, a}$, Yadong Zhang ${ }^{2}$ and Xiaolin Zuo ${ }^{1}$ \\ ${ }^{1}$ Henan University of Science \& Technology, 471003 Luoyang, China \\ ${ }^{2}$ Shangqiu University Applied Science and Technology College, 475000 Kaifeng, China
}

\begin{abstract}
In this paper, the working principle of the grating measurement system is combined with the Fourier analysis method of Moiré fringe to establish the mathematical model of the grating signal Lissajous figure to know the quality of the grating signal intuitively. The Mathematica numerical analysis software is used to obtain the graphics of the model, and the correctness of the relationship between the parameters of the grating measurement system and the Lissajous figure equation of the grating signal is verified. The influence of the grating pair angle $\alpha$ on the output voltage signal and Lissajous figure of the grating measurement system is studied. The results show that the intensity of the two-phase output electrical signal decreases gradually with the increase of the deviation of the angle $\alpha$ of the grating pair, but the equalamplitude of the two-phase output electrical signal does not change; Meanwhile, the shape of the grating signal Lissajous figure gradually changes from the ideal circle to the non-ideal ellipse, until a straight line with a strip slope of $135^{\circ}$ is formed.
\end{abstract}

\section{Introduction}

In the mechanical precision machining and testing, in order to achieve the expected effect of the design of the manufactured product, it is necessary to configure the measurement and detection device for real-time measurement and control of the product processing in the entire processing control system. The device should have the characteristics of good test stability, high precision, high efficiency, high resolution and easy to realize digitization and automation. The grating sensor is often used in measurement and detection devices to achieve the above requirements [1]. The standard signal output by the grating sensor measurement system is fourphase or two-phase sine wave or square wave with a phase difference of $90^{\circ}$ in turn, and the measurement accuracy of the system is closely related to the quality of waveform. If there are manufacturing, installation or motion errors in the grating measuring system, the output signal is disturbed, resulting in the output waveform deviating from the standard wave, thus affecting the measurement accuracy [2-4]. Therefore, it is of great significance to study the variation of output signal waveform of grating measuring system for improving the performance of grating sensor.

Mathematically, Lissajous figure can reflect the equal amplitude, orthogonality, sinusoidality, frequency ratio and phase difference of the two-phase signal, which reflect the quality of the two signals [5-6]. In the published literature and practical applications, the grating signal Lissajous figure is the most intuitive way to express the improvement of signal quality before and after correction, compensation and filtering, its acquisition method is to simulate two-phase sine or cosine signals assuming phase difference or to connect oscilloscope measurements with hardware circuits [7-9], without using the mathematical relationship in theory, the acquisition of the grating signal Lissajous figure has been fully studied from the point of view of the working principle of the grating measuring system. Therefore, it is especially important to study the relationship between the parameters of the grating measurement system and the Lissajous figure equation to judge the quality of the grating signal.

In this paper, the transmission grating sensor is taken as the research object, and the mathematical model of the grating signal Lissajous figure is established by using the working principle of the grating measuring system and the Fourier analysis method of the Moire fringe. The influence of different grating pair angle $\alpha$ on the output electrical signal and Lissajous figure of grating measuring system is studied. It is concluded that the phase difference of two-phase output electrical signal can be changed by adjusting the grating pair angle $\alpha$, so that the grating signal Lissajous figure is close to the circle and has the largest shape, so as to obtain highquality grating signal.

\section{Establishment of mathematical model of Lissajous figure}

In the grating measuring system, if the grating g1 has a grating pitch of $p$, the width of the transmissive slit is $w_{t}=p / 2$, and the transmittance of the transmissive slit is 1.

aCorresponding author: wxy2.0@163.com 
A grating rectangular coordinate system is set up in the plane of the grating g1, as shown in Figure 1. When the collimated light irradiates the grating $\mathrm{g} 1$, the transmission function $T_{1}(x)$ of the grating $\mathrm{g} 1$ is a rectangular wave function, and the expression is:

$$
T_{1}(x)=\left\{\begin{array}{l}
1, \text { transmission region, } k p \leq x \leq k p+\frac{w_{t}}{2} \\
0, \text { non-transmission region, else }
\end{array}\right.
$$

In the formula, $k$ is an integer.

Formula (1) is a periodic function whose Fourier expression is:

$$
T_{1}(x)=\sum_{n=-\infty}^{\infty} A_{n} e^{\left(\frac{i 2 \pi n x}{p}\right)}
$$

In the formula, $A_{n}=\frac{w_{t}}{p} \sin c\left(n \frac{w_{t}}{p}\right)$ is the Fourier coefficient, $n=0, \pm 1, \pm 2, \ldots$

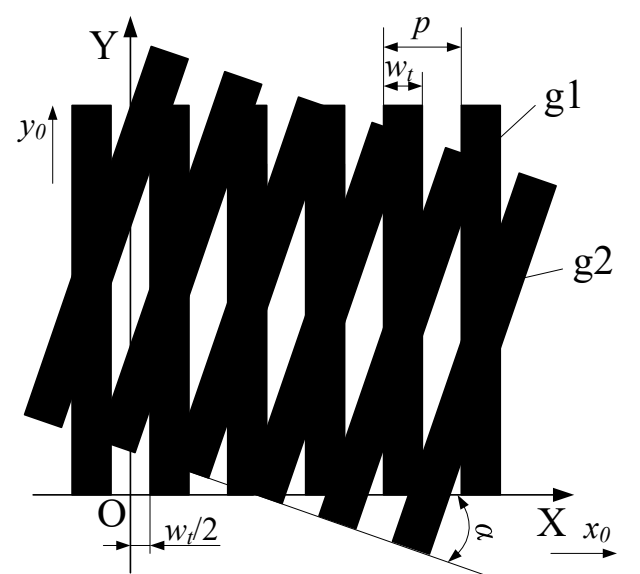

Figure 1. Grating rectangular coordinate system

The grating g2 in the grating measuring system is obtained by rotating the grating with the same parameters and positions as the grating $\mathrm{g} 1$ around its own plane by the $\alpha$ angle, and then the grating g2 is moved relative to the grating $\mathrm{g} 1$ along the $\mathrm{X}$-axis and the $\mathrm{Y}$-axis to generate displacements $x_{0}$ and $y_{0}$, as shown in Figure 1. According to the coordinate transformation, the Fourier expression of the transmission function of the grating g2 is:

$$
T_{2}\left(x-x_{0}, y-y_{0}\right)=\sum_{m=-\infty}^{\infty} B_{m} e^{\left\{i 2 \pi m \frac{\left[\left(x-x_{0}\right) \cos \alpha+\left(y-y_{0}\right) \sin \alpha\right]}{p}\right\}}
$$

In the formula, $B_{m}=\frac{w_{t}}{p} \sin c\left(m \frac{w_{t}}{p}\right)$ is the Fourier coefficient, $m=0, \pm 1, \pm 2, \ldots$

According to the theory of information optics and the conversion properties of Moiré fringes, the transmission function $T$ of Moiré fringe is the product of the transmission function $T_{1}(x)$ of the grating $\mathrm{g} 1$ and the transmission function $T_{2}(x)$ of the grating $\mathrm{g} 2$ when the grating $\mathrm{g} 1$ and the grating $\mathrm{g} 2$ are in relative motion along the $\mathrm{X}$-axis, and it has the following relations with Moiré fringe spacing $l$, namely:

$$
\begin{aligned}
T & =T_{1}(x) \times T_{2}\left(x-x_{0}, y-y_{0}+l\right) \\
& =\sum_{n=-\infty}^{\infty} \sum_{m=-\infty}^{\infty} A_{n} \times B_{m} \times e^{\left(\frac{i 2 \pi}{p}\right) \times\left\{n x+m\left[\left(x-x_{0}\right) \cos \alpha+\left(y-y_{0}+l\right) \sin \alpha\right]\right\}}
\end{aligned}
$$

Among them, the relationship between the Moiré fringe spacing $l$ and the grating pitch $p$ and the grating pair angle $\alpha$ is:

$$
l=\frac{p}{2 \sin \frac{\alpha}{2}} \approx \frac{p}{\alpha}
$$

Assuming that the incident light intensity is $L_{i n}$, the emergent light intensity $L_{\text {out }}$ of the obtained Moiré fringe after the light passes through the grating pair is:

$$
L_{\text {out }}=L_{\text {in }} \times T
$$

In the grating measuring system, the four phase optical system and the transverse Moiré fringe produced by the system are the most widely used. During the measurement, the intensity of the photoelectric element varies with the relative motion of the grating pair. The photoelectric element referred to herein is composed of four silicon photo cells $A_{1}, B_{1}, A_{2}$, and $B_{2}$, and is placed in a Moiré fringe space in turn. According to the principle of grating measurement, the size of the photosensitive surface of four silicon photocells is used as the integral interval respectively, and the intensity of the emergent light $L_{\text {out }}$ of the Moire fringe is taken as the integrand function, and the spacing $l$ of the Moire fringes is regarded as the integral variable in any period of the intensity of Moiré fringe emergent light. The integral operation can be used to obtain the intensity of the light received by each silicon photocell as a function of the displacement $x_{0}$, which is represented by $L_{A 1}\left(x_{0}\right), L_{B 1}\left(x_{0}\right)$, $L_{A 2}\left(x_{0}\right)$ and $L_{B 2}\left(x_{0}\right)$ respectively.

According to the principle of photoelectric conversion, assuming that the photoelectric conversion coefficients of each silicon photocell are $Q_{A 1}, Q_{B 1}, Q_{A 2}$ and $Q_{B 2}$, respectively, and the output voltage of each silicon photocell is the product of the light intensity received by each silicon photocell and the corresponding photoelectric conversion coefficients, expressed by $U_{A 1}\left(x_{0}\right), U_{B 1}\left(x_{0}\right), U_{A 2}\left(x_{0}\right)$ and $U_{B 2}\left(x_{0}\right)$, which is the voltage signal of the four-phase phase difference of $90^{\circ}$ outputted by the grating measuring system, and is approximately sinusoidal.

The A and B two-phase grating signals $\Delta U_{A}\left(x_{0}\right)$ and $\Delta U_{B}\left(x_{0}\right)$ with phase difference of $90^{\circ}$ can be obtained by differential processing of two voltage signals with phase difference of $180^{\circ}$ in $U_{A 1}\left(x_{0}\right), U_{B 1}\left(x_{0}\right), U_{A 2}\left(x_{0}\right)$ and $U_{B 2}\left(x_{0}\right)$ four-phase signals to reduce the DC component of the signal and improve the precision of Moiré fringe subdivision. That is, the parameter equation of the grating signal Lissajous figure about the displacement $x_{0}$, and its expression is:

$$
\left\{\begin{array}{l}
\Delta U_{A}\left(x_{0}\right)=U_{A 1}\left(x_{0}\right)-U_{A 2}\left(x_{0}\right) \\
\Delta U_{B}\left(x_{0}\right)=U_{B 1}\left(x_{0}\right)-U_{B 2}\left(x_{0}\right)
\end{array}\right.
$$

\section{Example Calculation and Analysis}

\subsection{Calculation Model}

Taking a pair of grating pairs with pitch $p=0.02 \mathrm{~mm}$, transmission slit width $w_{t}=p / 2=0.01 \mathrm{~mm}$, and angle $\alpha=0.1^{\circ}$ as an example [10], the grating signal Lissajous 
figure is simulated. Substituting the pitch $p=0.02 \mathrm{~mm}$ and angle $\alpha=0.1^{\circ}$ into the formula (5), $l=11.4592 \mathrm{~mm}$ can be obtained by calculation. The incident light intensity is assumed to be $5 \mathrm{~cd}$. According to the formula (6), the relationship between the Moiré fringe spacing $l$ and the emergent light intensity $L_{\text {out }}$ can be obtained, as shown in Figure 2 .

\section{L cd}

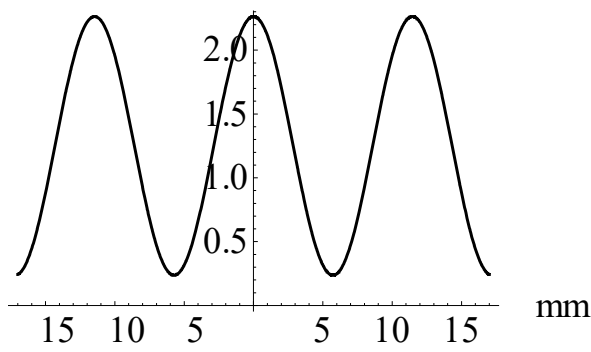

Figure 2. About the emergent light intensity $L_{\text {out }}$ distribution diagram of the Moiré fringe spacing $l$

For the convenience of calculation, the four silicon photo cells are sequentially placed on the first period to the right of the ordinate of the Moire fringe emergent light intensity distribution diagram shown in Figure 2, The integral operation is carried out with the Moiré fringe spacing $l$ as the integral variable, the Moire fringe emergent light intensity $L_{\text {out }}$ as the integrand function, and the size of the photosensitive surface of four silicon photo cells as the integral interval. Ideally, the size of the photosensitive surface of the four silicon photocells is equal to the spacing $l$ of one Moiré fringe. It can be calculated that the light intensity distribution of the four silicon photocells with respect to the displacement $x_{0}$ is as shown in Figure 3.

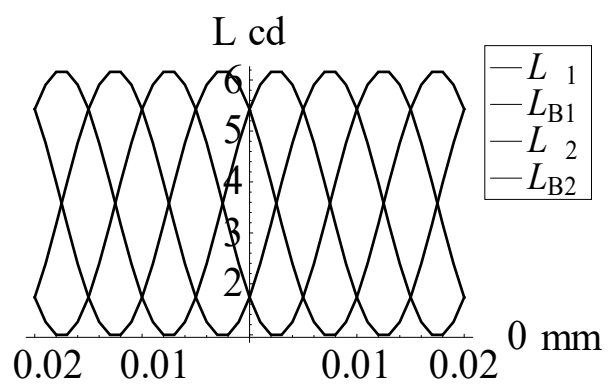

Figure 3. Light intensity distribution diagram of silicon photocells $A_{1}, B_{1}, A_{2}$ and $B_{2}$

In this study, the photoelectric conversion coefficient $Q_{A 1}=Q_{B 1}=Q_{A 2}=Q_{B 2}=1.2$ is taken, and multiplied by the light intensity $L_{A 1}\left(x_{0}\right), L_{B 1}\left(x_{0}\right), L_{A 2}\left(x_{0}\right)$ and $L_{B 2}\left(x_{0}\right)$ of the four silicon photocells respectively. The output voltage curves of the four silicon photocells with respect to displacement $x_{0}$ can be obtained, as shown in Figure 4 .

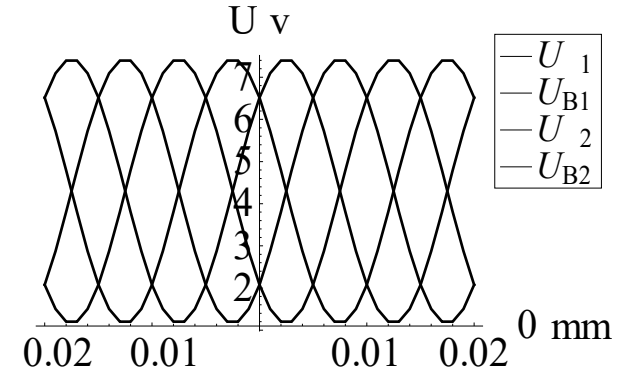

Figure 4. Voltage distribution diagram of silicon photocells $A_{1}$, $\mathrm{B}_{1}, \mathrm{~A}_{2}$ and $\mathrm{B}_{2}$

It can be seen from Figure 4 that the electrical signals output by the four silicon photovoltaic cells are sequentially different by $90^{\circ}$ in phase, and the distribution are approximately sinusoidal. The signal of phase difference of $180^{\circ}$ in two phases in Figure 4 is subjected to differential processing to obtain the A and $\mathrm{B}$ two-phase voltage signals $\Delta U_{A}\left(x_{0}\right)$ and $\Delta U_{B}\left(x_{0}\right)$ with phase difference of $90^{\circ}$, that is, the voltage difference curves of two-phase signals of A and B with respect to displacement $x_{0}$, as shown in Figure 5.

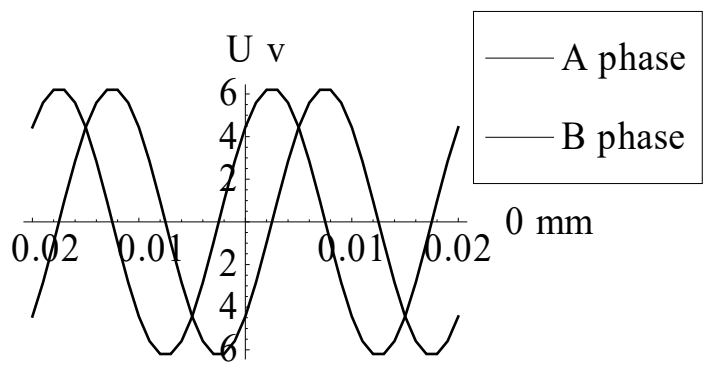

Figure 5. Voltage difference waveforms of A and B two-phase

The abscissa and ordinate of the grating signal Lissajous figure are the A phase signal and the B phase signal respectively in Figure 5, as shown in Figure 6. The grating signal Lissajous figure is the standard circle consistent with the ideal grating signal Lissajous figure.

$\mathrm{U} v \mathrm{~B}$ phase

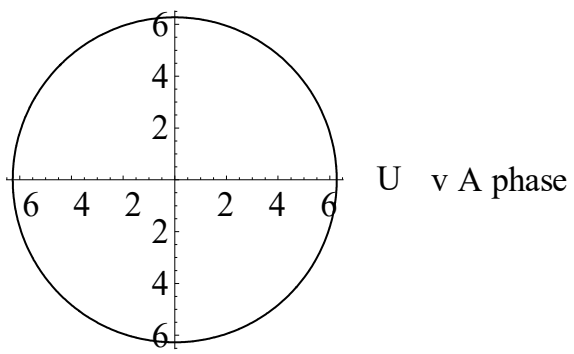

Figure 6. $\alpha=0.1^{\circ}$, Lissajous figure of grating signal

\subsection{The voltage signal and Lissajous figure corresponding to the different grating pair angle}

The different voltage signals can be obtained by adjusting the parameters of Lissajous figure mathematical model of grating signal, and then the shape of the grating signal Lissajous figure can be affected. When different angles $\alpha$ of grating pairs are taken in the mathematical model of the grating signal Lissajous figure, the output voltage signal and the corresponding 
Lissajous figure of the grating measuring system are shown in Figure 7.
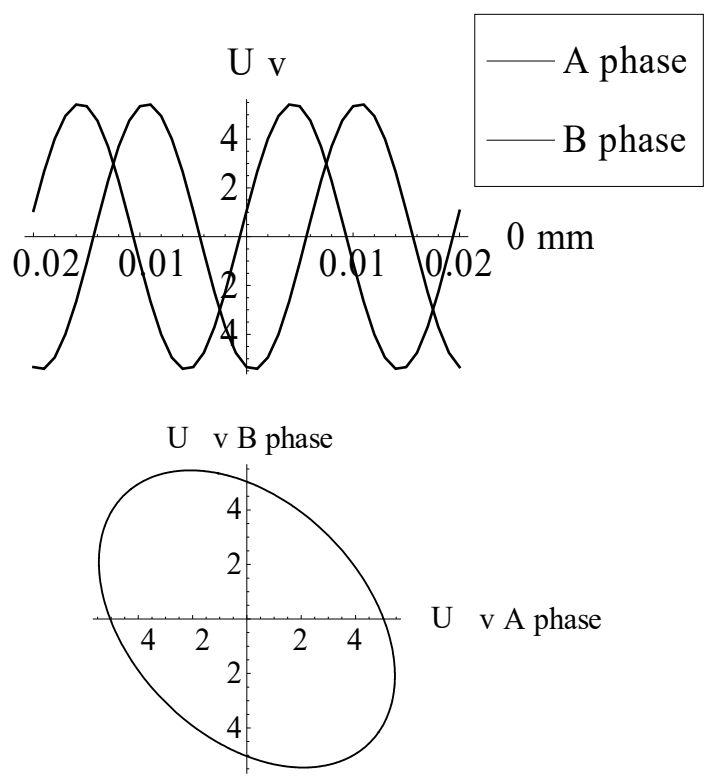

a) $\alpha=0.125^{\circ}$
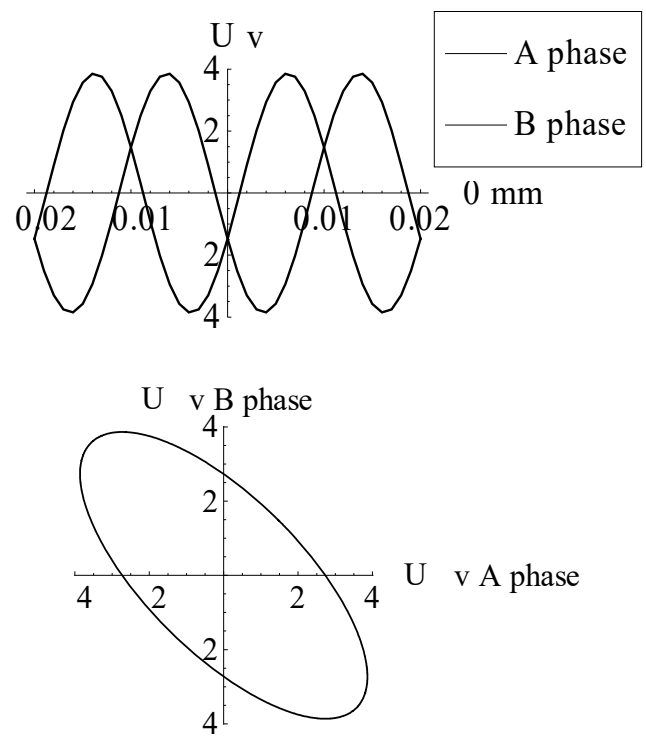

b) $\alpha=0.15^{\circ}$

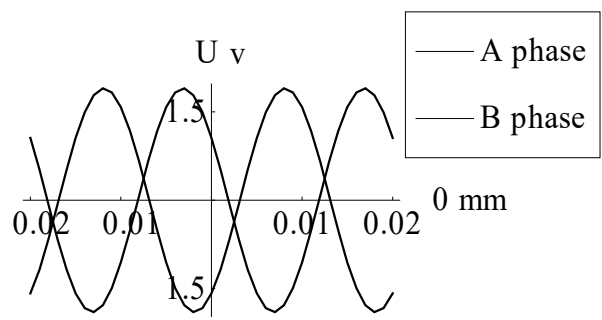

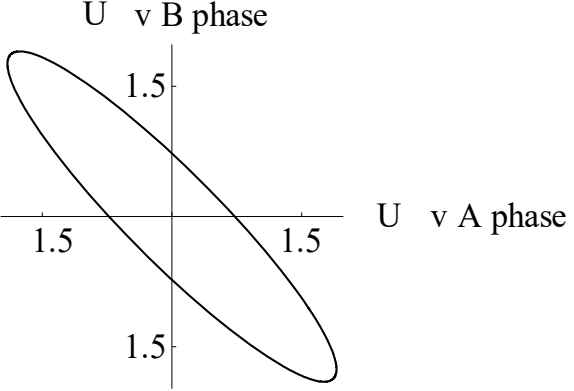

c) $\alpha=0.175^{\circ}$

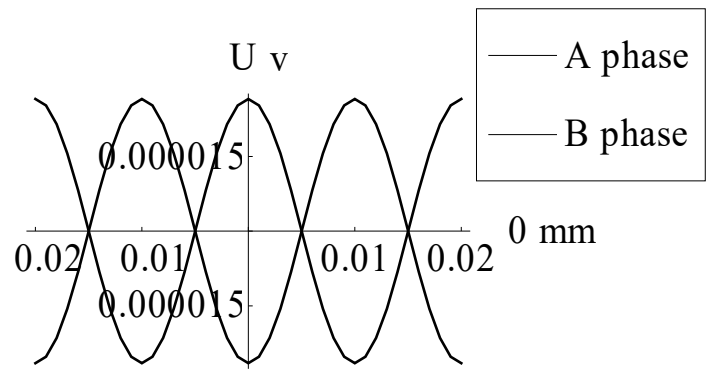

U v B phase

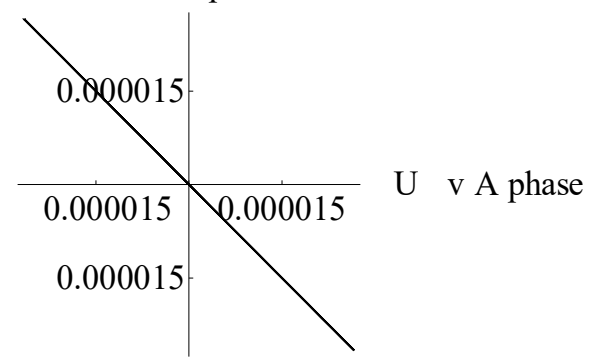

d) $\alpha=0.20^{\circ}$

Fig. 7 The voltage signal and Lissajous figure corresponding to the different $\alpha$

It can be seen from the above Figure 7 that when the values of the grating pair $\alpha$ angle are different, the equal amplitude of the two-phase voltage signal output by the grating measuring system remains unchanged, but the greater the deviation of the $\alpha$ angle of the grating pair, the lower the intensity of the output signal, and the smaller the shape of the corresponding grating signal Lissajous figure; On the other hand, the phase difference of the two-phase voltage signal is no longer maintained at $90^{\circ}$ with the deviation of the $\alpha$ angle of the grating pair, and the shape of the corresponding grating signal Lissajous figure changes from the original circle to ellipse, and the shape of the ellipse becomes more and more flat, and the direction of the ellipse changes with the phase angle. Until the two-phase voltage signal has no phase difference, the grating signal Lissajous figure is shaped as a straight line with a slope of $135^{\circ}$.

The flatter the shape of the grating signal Lissajous figure, the lower the precision of subdivision, and the lower the accuracy of the grating measurement. According to the above analysis, the flat grating signal Lissajous figure can achieve high-precision and highfrequency subdivision of the grating signal by adjusting the $\alpha$ angle of the grating pair to make it close to circle. Therefore, in order to obtain high-precision 
measurement results in practical use, it is necessary to reduce the extent to which the grating pair $\alpha$ angle deviates from the ideal value as much as possible to obtain near-circular Lissajous figure and maintain the intensity of the grating measurement output signal.

\section{Conclusion}

The establishment of the mathematical model of the grating signal Lissajous figure can conveniently and efficiently obtain the corresponding grating signal Lissajous figure with different output voltage signals. By studying the mathematical model of grating signal Lissajous figure and the output voltage signal and the Lissajous figure corresponding to the different grating pair angle $\alpha$, the following conclusions are drawn:

(1) The mathematical model of the grating signal Lissajous figure is established, which provides a theoretical basis for obtaining the grating signal Lissajous figure.

(2) Changing the angle $\alpha$ of the grating pair cannot change the equal amplitude of the two-phase output electrical signal, but can simultaneously change the amplitude of the two-phase output electrical signal and the size of the Lissajous figure.

(3) Changing the angle $\alpha$ of the grating pair can change the phase difference of the two-phase output electrical signal, so that the shape of the grating signal Lissajous figure is gradually changed from the ideal circle when the phase difference of the two-phase output electrical signal is $90^{\circ}$ to the non-ideal ellipse until a straight line with slope of $135^{\circ}$ is formed when the phase difference is $180^{\circ}$.

(4) The unsatisfactory Lissajous figure can be adjusted to the ideal Lissajous figure by appropriately adjusting the angle $\alpha$ of the grating pair to provide a method for improving the quality of the grating signal.

(5) The research provides theoretical guidance for designing, manufacturing, installing and debugging grating pairs scientifically; it provides a theoretical foundation for further studying the error of grating measurement signals.

\section{Acknowledgement}

In this paper, the research is supported by the National Natural Science Foundation of China (Project No. 51775172).

\section{References}

1. Zhao Guijuan. Application of Grating Sensor in Numerically-controlled Machine Tool[J]. Coal Mine Machinery, 2009, 30(6):171-172.

2. Feng Chaopeng, Zhu Lianqing, Pan Zhikang, et al. New Self-calibration Method of Circular Grating Eccentric Parameters[J]. Chinese Journal of Scientific Instrument, 2016, 37(11):2459-2464.

3. Chen Xiaohuai, Du Guoshan, Cheng Zhenying. Research on Dynamic Error of Grating
Measurement System[J]. Journal of Electronic Measurement and Instrument, 2012, 26(3):187-191.

4. Guo Xiaocong, Niu Yongsheng, Zhao Huiying, et al. Analysis on Several Factors Affected the Accuracy of Grating Sensors[J]. Manufacturing Automation, 2015(3):71-73.

5. Wang Xianjun. Errors and Precision Analysis of Subdivision Signals for Photoelectric Angle Encoders[J]. Optics and Precision Engineering, 2012, 20(2):379-386.

6. Ye Shuliang, Zhang Qian, Zhu Weibin. Study on Quadrature Error Real-time Compensation for Grating Moiré Signal[J]. Chinese Journal of Scientific Instrument, 2017, 38(1):57-64.

7. Guo Yumei, Lu Hongbo. Non-sinusoidal Error Correction for Grating Moiré Fringe Signal[J]. Journal of Shenyang University of Technology, 2015, 37(6):679-683.

8. Gao Xu, Wan Qiuhua, Zhao Changhai, et al. Realtime Compensation of Orthogonality Deviation for Moiré Fringe Photoelectric Signal[J]. Optics and Precision Engineering, 2014, 22(1):213-219.

9. Wu Hongsheng, Zeng Qifeng, Qiao Dong, et al. Filtering Method of Improving Quality of Grating Moiré Fringe[J]. Optics and Precision Engineering, 2011, 19(8):1944-1949.

10. Zhang Shanzhong. Measuring Grating Technique[M]. Beijing:China Machine Press, 1985. 